\title{
Effect of using adaptive seating equipment on grasping and visual motor integration in children with hemiparetic cerebral palsy: a randomized controlled trial
}

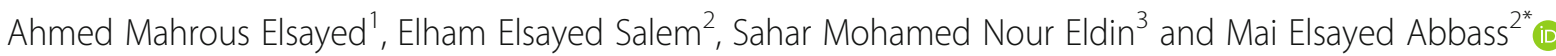

\begin{abstract}
Background: Adaptive seating is commonly used as an intervention method to enhance postural control. The aim of this study is to investigate the effect of using therapy ball as a seat alternative to using typical chair on grasping and visual motor integration in the children with hemiparetic cerebral palsy. For this aim, thirty children with hemiparetic cerebral palsy from both sexes were included in this study. The children ages ranged from 3 to 6 years old. The degree of spasticity was 1 to $1+$ according to modified Ashworth scale. The children were able to sit independently and follow instructions. Children were randomly assigned into two groups (experimental group and control group). Each child was evaluated before and after 3 successive months of selected occupational therapy exercises program. All the children of both groups received the same selected occupational therapy exercises program, but the children in the experimental group performed the exercises while sitting on therapy ball, and the children in the control group performed the exercises while sitting on typical chair.
\end{abstract}

Results: There was a significant improvement in the measured variables for both groups after treatment. The posttreatment results of the two groups of grasping and visual motor integration for age equivalent scores revealed significant difference ( $p=0.008$ and $p=0.011$ respectively) in favor of the experimental group.

Conclusions: Therapy ball could be used as a seat alternative to using typical chair to facilitate visual motor integration and grasping in the children with hemiparetic cerebral palsy.

Keywords: Cerebral palsy, Spastic, Rehabilitation, Hand, Visual motor coordination

\section{Background}

Dysfunction of the upper extremity is a common disabling consequence of cerebral palsy particularly in hemiplegic cerebral palsy. Common disorders which affect upper extremity function include spasticity, weakness, limited forearm supination, and muscle shortening. These impairments lead to problems in reaching, grasping, releasing, and manipulating objects [1].

\footnotetext{
* Correspondence: mai.tamer.elmasry@gmail.com

${ }^{2}$ Department of Pediatric Physical Therapy, Faculty of Physical Therapy, Cairo University, El-Tahrir st. - in front of Ben El-Sarayat Traffic, Dokki, Giza 12613, Egypt

Full list of author information is available at the end of the article
}

Postural control involves orientation of the body in space to provide a stable base for function and task performance. Proper postural control is fundamental for execution of controlled coordinated and symmetrical movement of the upper extremity [2].

In the children with cerebral palsy, the dysfunction in the ability to coordinate trunk muscles in the proper sequence leads to postural instability and affects the performance of the upper limbs [3]. Stimulating proper control of posture results in better functional execution of activities. The children with hemiparesis benefit from the modification of seating surface to improve postural control and the quality of upper limb reaching [4]. 
Adaptive seating devices help to maintain head and trunk stability. Physical therapists are involved in the rehabilitation of individuals with multiple handicaps frequently recommend a suitable chair to facilitate functional position, to increase sitting comfort and to improve performance of manipulative skills [5].

Adaptive seating is commonly used as an intervention method to enhance postural control in the children with cerebral palsy. These adaptive seating devices have the ability to improve the physical function. This improvement is maintained even after the end of the intervention [6].

Since 1991, there has been an increasing information about the benefits of using balls as an adaptive seating to replace chairs in schools of Europe. It was found that active seating has many benefits in preventing back injury, improving poor hand writing, helping hyperactive children to be calmer, and focusing for longer time as well as helping other children to concentrate better with better understanding of subject materials [7]. Therapy balls as an alternative seating device help to facilitate positive classroom behaviors, mental health, and fitness [8].

The previous studies are focusing on using therapy balls as a replacement for chairs to improve posture, back health, and school performance. In Switzerland where Swiss balls originated, they have a program called "Moving students are better learners." This program has the philosophy that moving students become less bored and having the ability to focus more on classroom activities. It helped the children to move from place to another without moving the furniture and causing noise. This makes the classroom environment calmer and helps the children to concentrate better [9].

Therapy balls also were used as a seat alternative to using office chairs. They helped to maintain active seating posture to maintain the balance during sitting on the ball as it keeps the abdominal and back muscles active [10].

Therapy ball chairs are increasingly used with children's sensory and motor impairments. It has the benefits of improving balance, addressing postural control, attention, and improving vestibular and proprioceptive sense [11].

There are a few studies applied to assess the effect of using therapy ball as a seat alternative to using chairs in handicap children. The focus was on its effect on behavior and participation in the special education classroom. The majority of these studies were applied on attention deficit hyperactivity disorder $[8,12-14]$ and autism spectrum disorder $[9,11]$. Also, other studies were done on normal students to assess the effect of therapy ball seating on sitting discomforts, task behavior, and academic achievement $[8,10,15]$.
The aim of this study is to investigate the effect of using therapy ball as a seat alternative to using typical chair on grasping and visual motor integration in children with hemiparetic cerebral palsy.

\section{Methods}

\section{Study design}

A randomized controlled trial. Children were assigned randomly (simple randomization; closed envelopes) into two groups with fifteen children in each group (experimental group and control group). The first author generated the randomization. This study adheres to CONSORT guidelines.

\section{Participants}

Thirty children with hemiparetic cerebral palsy were included in this study. Both sexes were considered. The age of the children was from 3 to 6 years old. All the parents of the children were informed about the purpose of the study and provided their written consent before participating in this study.

Inclusion criteria: Children were included in this study if they were diagnosed as children with hemiparetic cerebral palsy, the age was from 3 to 6 years old, and the degree of spasticity was from 1 to $1+$ according to modified Ashworth's scale [16]. They were able to sit independently and to follow instructions.

Exclusion criteria: Children were excluded if they had visual or auditory defects that may affect their performance, had fixed upper limb deformities that hinder the assessment and training procedures, or had an injection with botulinum toxins in the last 6 months before enrollment.

As represented in Fig. 1, children were assigned randomly (simple randomization; closed envelopes) into two groups with fifteen children in each group (experimental group and control group). Evaluation was carried out before and after three successive months, 3 times/ week for $1 \mathrm{~h}$ for the experimental and the control groups. All the children of both groups received the same selected occupational therapy exercises program but the children in the experimental group performed the exercises while sitting on therapy ball seating and the control group performed the exercises while sitting on typical chair. Intervention was conducted and data was collected in the outpatient clinic of Faculty of physical therapy, Cairo University.

\section{Materials}

\section{For the evaluation of grasping and visual motor} integration

Peabody developmental motor scal $2[17,18]$ is used. It is a norm-referenced gross and fine motor scale that is used for the evaluation of motor development in the 


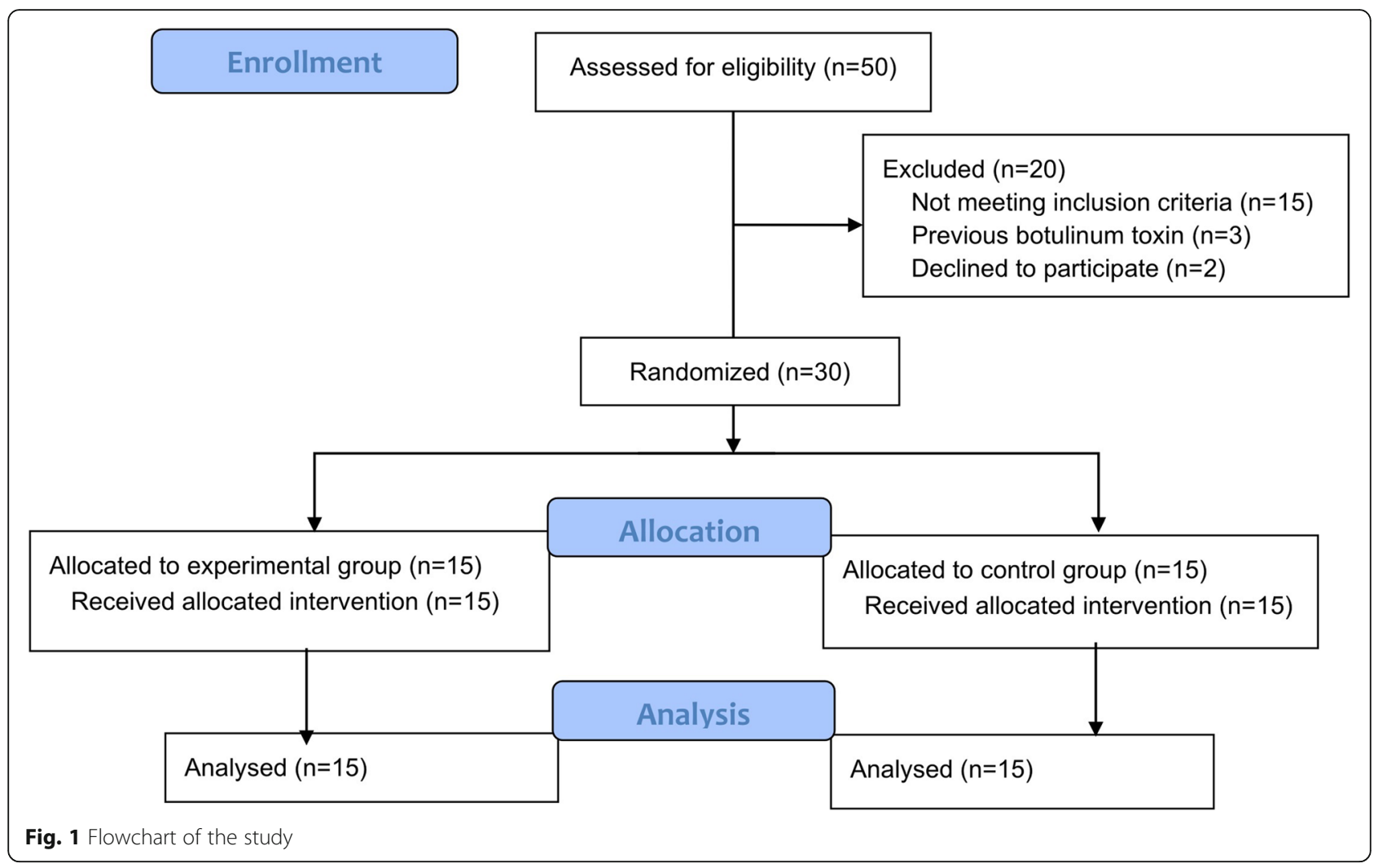

children with motor development delay. The scale consists of 98 fine motor items which are divided into two subtests (26-item grasping and 72-item visual-motor integration). It has an excellent validity and reliability as a discriminative measure $[17,18]$. Separate scores could be obtained for visual-motor integration and grasping to assess the ability of the child to perform eye-hand coordination tasks and to use his or her hands respectively. The testing scores could be represented as standard scores or age equivalent scores which are used in this study to assess the improvement in fine motor abilities of the children and the improvement in their developmental age.

\section{For the treatment}

For the experimental group: different sizes of therapy balls were used as an adaptive seating equipment during intervention according to the child height. Adjustable rubber ring was placed under the ball to increase its stability. The child should sit on the ball with right angles at the hip and the knee joints with his or her feet rested on the ground. The child sat in front of a table adjusted to his or her height to allow right angles of the elbows while the forearms rested on the table.
For the control group: Wooden armless chairs with different heights and sizes according to the child height were used during intervention. The child should sit on the chair with right angles at the hip and the knee joints with his or her feet rested on the ground. The child sat in front of a table adjusted to his or her height to allow right angles of elbows while the forearms rested on the table.

For both experimental and control groups: the following tools were used to administer the selected exercises program: shoelace, card with holes, hair brushes, rattles, scissors, bottles with screw-on caps, large button strip, cups and spoons, cubes, puzzle, sheets, markers, toothbrushes, toys, penny, sponge, and different sized beads.

\section{Procedures}

For the evaluation of grasping and visual motor integration

Test scoring: Peabody developmental motor scale 2 [17, 18 ] is applied as follows. The child scored 2 if the testing item was performed according to the criteria specified for mastery. The child scored 1 if the testing item was performed with a clear resemblance to the item mastery criteria but did not fully meet the criteria. The child scored zero if he or she could not attempt the item. 
Test administration: Entry/start point, basal level and ceiling level should be used for all subtests:

Item administration begins at the point appropriate for the child's age (entry point). The child should receive a score of (2) on the first 3 consecutive items to go forward. If the child scored (0) or (1) on any of the first 3 items conducted starting from the entry point, we tested backward until the child scored 2 on three items in a row (basal level). Once the basal has been established, the examiner administered progressively more difficult items until a ceiling is established. The ceiling is determined when the child scored (0) on each of 3 items in a row (ceiling level). A child was given a maximum of 3 trials for each item before scoring. Each item was given a score of (0), (1), or (2). The child's total raw score for each subtest was computed by adding the total score for all items in the subtest.

Interpreting the results: The scale has 5 types of scores: raw scores, percentiles, standard, age equivalent scores for the subtests, and quotients for the composites. The raw scores calculated from each subtest, the standard scores and the age-equivalent scores are obtained from the norm's tables provided in the manual.

\section{For the treatment}

Children were assigned randomly (simple randomization; closed envelopes) into two groups with fifteen children in each group (experimental group and control group). All the children of both groups received the same selected occupational therapy exercises program but the children in the experimental group perform the exercises while sitting on therapy ball seating and the control group perform the exercises while sitting on chair.

Each exercise was explained and presented first by the therapist, then the child was allowed to perform the task by him or herself with continuous instruction and correction administered by the therapist. The whole intervention session was $1 \mathrm{~h}$ long for each child. The study design did not include the collection of any information on harms

The following purposeful tasks were used: inserting different shapes in the correct hole, stringing beads, copying square and triangle, dropping pellets, folding and crumpling paper, closing and opening a bottle, using scissors to cut a paper, reaching above the level of shoulder, tracing line and connecting dots, reaching across the midline, buttoning and unbuttoning button, squeezing water out of a sponge, clapping, lacing string, turning book's pages, using fine finger movements to pick up a small piece of food, removing socks, manipulating a penny from fingers to palm and vice versa, using a toothbrush, and using colorful blocks with different textures and weights to build towers.

\section{Statistical analysis}

Statistical analysis of the results was performed through the statistical package for social sciences (SPSS) version 25 for windows. Wilcoxon matched signed rank-test was used to evaluate the differences between the results before and after the treatment program within each group (experimental and control groups). Mann-Whitney test was used to evaluate the differences between post treatment program results for the experimental and control groups. The level of significance for all statistical tests was set at $p<0.05$.

\section{Results}

Thirty spastic children with hemiparetic cerebral palsy were included in this study. They were randomly assigned into two groups (experimental and control groups) with 15 children (6 females, 11 left hand affected) in the control group and 15 children (5 females, 9 left hand affected) in the experimental group. The age of the children in the control and experimental groups ranged from 3 to 6 years old with mean range in months $51 \pm 10.2$ and $50.53 \pm 10.51$ respectively. Data obtained from both groups before and after 3 successive months of intervention was statistically treated to analyze the scale scores including grasping subtest standard scores, grasping subtest age equivalent, visual motor integration subtest standard score, and visual motor integration subtest age equivalent. Comparing the results of age in months of the children of both groups revealed that there were no significant differences found between both groups in the chronological age $(p=0.903)$.

As presented in Table 1, the comparison between the data collected from both groups before the beginning of the treatment revealed that there was no significant difference between both groups.

As presented in Table 2, the comparison between pretreatment and post-treatment results of the control group revealed that there was significant difference between the results.

As presented in Table 3, the comparison between pretreatment and post-treatment results of the experimental group revealed that there was significant difference between the results.

As presented in Table 4 and Fig. 2, the comparison between the results of both groups after treatment revealed that there was significant difference between both groups in all results.

\section{Discussion}

This study was conducted to investigate the effect of using therapy ball as a seat alternative to using typical chair on grasping and visual motor integration in the children with hemiparetic cerebral palsy. For this purpose, 30 children with hemiparesis participated in this 
Table 1 Comparison of pre-treatment results of experimental and control groups

\begin{tabular}{|c|c|c|c|c|}
\hline & Control group (median and IQR) & Experimental group (median and IQR) & $P$-value & Sig. \\
\hline Grasping subtest (standard score) & $1(1-1)$ & $2(1-2)$ & 0.101 & NS \\
\hline Grasping subtest (age equivalent score) & $9(6-10)$ & $9(7-14)$ & 0.628 & NS \\
\hline Visual motor integration (standard score) & $3(2-3)$ & $2(2-3)$ & 0.335 & NS \\
\hline Visual motor integration (age Equivalent score) & $14(12-18)$ & $13(11-16)$ & 0.585 & NS \\
\hline
\end{tabular}

IQR interquartile range, NS non-significant, $P$-value probability value, Sig. significance

study. All the children were assessed before starting the intervention and immediately after the last treatment session by Peabody Developmental Motor Scales $2[17,18]$.

All the pre-treatment results revealed that there was no significant difference between the children of the experimental and the control groups which demonstrates the homogeneity between both groups before the starting of the study.

The chronological ages of the children in the control and the experimental groups in months were $51 \pm 10.2$ and $50.53 \pm 10.51$ respectively. However, the assessment results of their developmental age before intervention revealed that they had developmental delay in their grasping, visual motor integration abilities as the developmental ages of the children in the control group were (9 (6-10) and 14 (12-18)) respectively, and the experimental group were $(9(7-14)$ and $13(11-16))$ respectively. The children found difficulties in bilateral activities, in movements which need fine finger movements and grip strength like grasping pellets, markers, touching fingers, removing pegs, imitating strokes, and using tools like scissors and spoons. This agrees with the results of the study by Sakzewski et al. [19] who studied the relation between unilateral manual capacity and bilateral performance. They concluded that there was a strong relationship between unimanual capacity and bimanual performance $(r=0.83)$. Also, this study mentioned that hemiplegic cerebral palsy had difficulties in achieving bilateral such as using knife, fork, and tying shoe laces [19].

The delayed development of grasping and visual motor integration activities might be as a result of decreased muscle strength and motor control of the upper limb of the hemiparetic children which affect performing tasks properly and interfere with the children development [20]. The movement of the upper limbs of the child is asymmetric and the affected limb is slower than the non-affected arm with a delay in the movement initiation [21].

After intervention, the children of the experimental and the control groups improved significantly in their age equivalent scores in grasping and visual motor integration subtest scores $(P$ value $=0.001,0.001$ respectively for the experimental group) and ( $P$ value $=0.001,0.001$ respectively for the control group). This significant improvement may be as a result of the children in the preschool age could progress rapidly in hand function development with intervention at this age. This comes in agreement with Hanna [22] and Fedrizzi et al. [23] who concluded that the hand function development in the children with cerebral palsy progresses during the preschool period, but stabilizes or even decreases as the children develop. Also these results come in agreement with Azzam [24] who studied the effect of an occupational therapy program on the hand function improvement in the children with hemiparesis (children age range from 4 to 6 years old) and concluded that the children in the experimental group improved significantly in their hand grip $(P=0.001)$.

The post-treatment results reflect the efficacy of the selected program for hand functions in form of play activities from functional position instead of isolated tasks without meaning. This agreed with Volman et al. [21] who studied the effect of task context intervention program on the quality of reaching, and concluded that functional relevant tasks may enhance the reaching quality than non-functional tasks.

Table 2 Comparison of pre-treatment and post-treatment results of the control group

\begin{tabular}{|c|c|c|c|c|}
\hline & Pre-treatment (median and IQR) & Post-treatment (median and IQR) & $P$-value & Sig. \\
\hline Grasping subtest (standard score) & $1(1-1)$ & $3(2-5)$ & 0.006 & S \\
\hline Grasping subtest (age equivalent score) & $9(6-10)$ & $18(11-34)$ & 0.001 & S \\
\hline Visual motor integration (standard score) & $3(2-3)$ & $3(3-3)$ & 0.008 & $S$ \\
\hline Visual motor integration (age Equivalent score) & $14(12-18)$ & $18(14-22)$ & 0.001 & S \\
\hline
\end{tabular}

IQR interquartile range, $P$-value probability value, $S$ significant, Sig. significance 
Table 3 Comparison of pre-treatment and post-treatment results of the study group

\begin{tabular}{|c|c|c|c|c|}
\hline & Pre-treatment (median and IQR) & Post-treatment (median and IQR) & $P$-value & Sig. \\
\hline Grasping subtest (standard score) & $2(1-2)$ & $7(6-8)$ & 0.001 & $S$ \\
\hline Grasping subtest (age equivalent score) & $9(7-14)$ & $37(22-43)$ & 0.001 & $S$ \\
\hline Visual motor integration (standard score) & $2(2-3)$ & $4(4-4)$ & 0.001 & $S$ \\
\hline Visual motor integration (age Equivalent score) & $13(11-16)$ & $24(21-26)$ & 0.001 & $\mathrm{~S}$ \\
\hline
\end{tabular}

$I Q R$ interquartile range, $P$-value probability value, $S$ significant, Sig. significance

Table 4 Comparison of post-treatment results of experimental and control groups

\begin{tabular}{|c|c|c|c|c|}
\hline & Control group (median and IQR) & Experimental group (median and IQR) & $P$-value & Sig. \\
\hline Grasping subtest (standard score) & $3(2-5)$ & $7(6-8)$ & 0.007 & $\mathrm{~S}$ \\
\hline Grasping subtest (age equivalent score) & $18(11-34)$ & $37(22-43)$ & 0.008 & $S$ \\
\hline Visual motor integration (standard score) & $3(3-3)$ & $4(4-4)$ & 0.005 & $\mathrm{~s}$ \\
\hline Visual motor integration (age Equivalent score) & $18(14-22)$ & $24(21-26)$ & 0.011 & $\mathrm{~S}$ \\
\hline
\end{tabular}

$I Q R$ interquartile range, $P$-value probability value, $S$ significant, Sig. significance

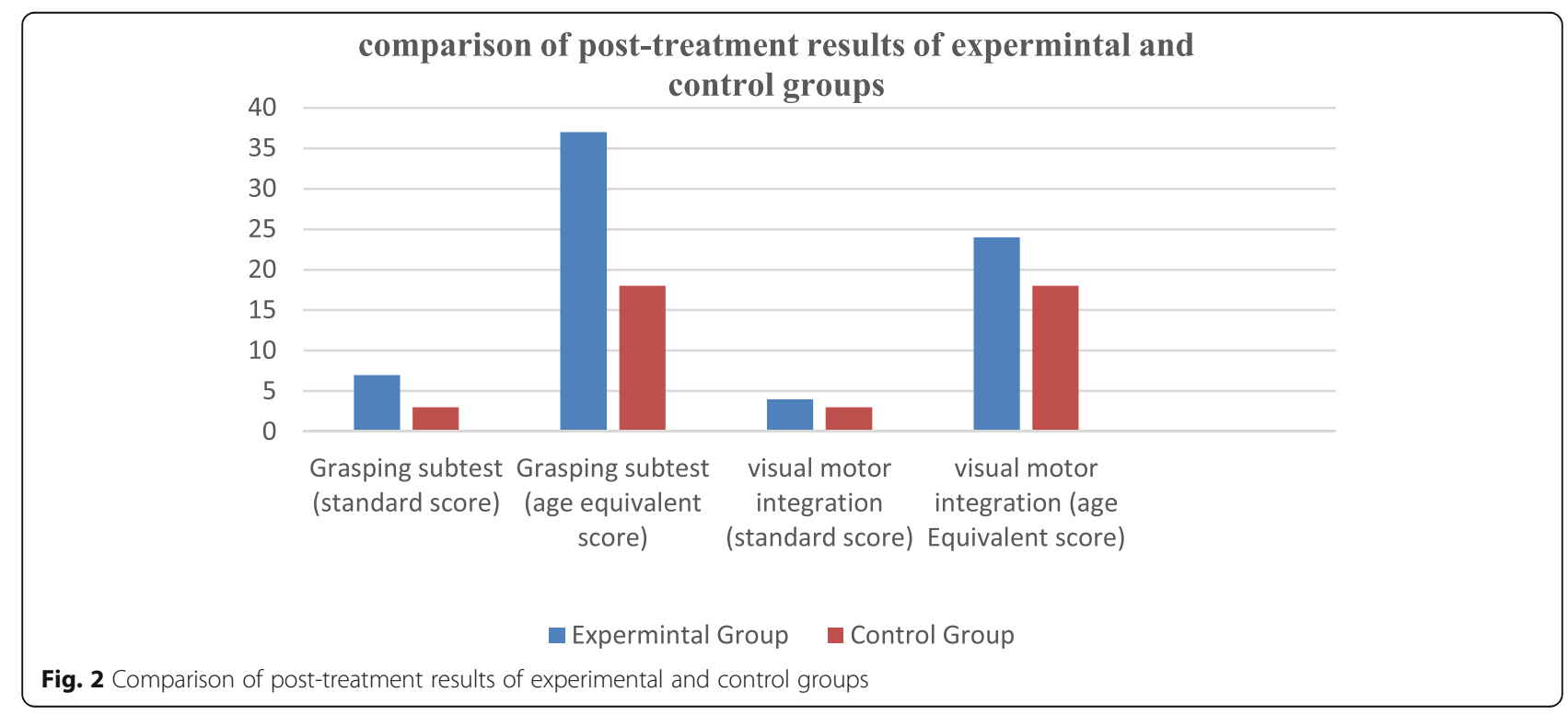


When comparing between post-treatment results of the experimental and the control groups, there was significant difference in the post-treatment results in favor of the experimental group in grasping and visual motor integration subtest scores $(P<0.05)$

This significant improvement in post-treatment results of the experimental group may be attributed to the use of adaptive therapy ball seating which encouraged children to improve their performance of the selected training tasks through maintaining active sitting. Good erect posture helped to provide a stable base for efficient hand function activities. Also, sitting on ball as an alternative to chair helped to reduce sitting discomfort and improved the concentration of the children on the task performance.

This agreed with Al-Eisa et al. [15] who studied the effect of using therapy ball as an adaptive seating equipment on the academic performance and sitting discomfort. They concluded that the sitting discomfort and academic performance were improved in the study group when compared to the control group using the typical classroom chairs.

Previous studies conducted on the effects of therapy balls in the school-age children instead of typical chairs focused mainly on the special education students, including students with attention deficit hyperactivity disorder [12-14] and the children with autism spectrum disorder [9, 11]. These studies concluded that using therapy ball in the classroom has positive effects on attention, participation, and in-seat behavior [9, 11-14].

The limitation of this study is the small number of the included sample so the results cannot be generalized. From this study, it is concluded that therapy ball could be used as an alternative for typical chair seating to facilitate grasping and visual motor coordination in children with hemiparetic cerebral palsy. It is recommended to conduct studies with larger samples and studies to investigate the effect of therapy ball on postural control and attention in the children with cerebral palsy.

\section{Conclusion}

Therapy ball could be used as an alternative for typical chair seating to facilitate grasping and visual motor integration in the children with hemiparetic cerebral palsy.

\section{Acknowledgements}

Not applicable

\section{Authors' contributions}

A M E and M E A conceived and designed the experiments; analyzed and interpreted the data; contributed reagents, materials, analysis tools or data; and wrote the paper. A M E performed the experiments. E E S conceived and designed the experiments and analyzed and interpreted the data. S M N conceived and designed the experiments. The authors read and approved the final manuscript.
Funding

No funding

Availability of data and materials

The datasets generated during and/or analyzed during the current study are available from the corresponding author on reasonable request.

\section{Declarations}

Ethics approval and consent to participate

This study was approved by the ethical committee of Faculty of Physical therapy under the number "REC/012/001765" and registered in ClinicalTrial. gov under the number "NCT04651283". Parents of all participants signed a written informed consent before starting the study.

\section{Consent for publication}

Not applicable

\section{Competing interests}

The authors declare that they have no competing interests.

\section{Author details}

${ }^{1}$ Sidi Salem General Hospital, Sidi Salem, Egypt. ${ }^{2}$ Department of Pediatric Physical Therapy, Faculty of Physical Therapy, Cairo University, El-Tahrir st. - in front of Ben El-Sarayat Traffic, Dokki, Giza 12613, Egypt. ${ }^{3}$ Medical Genetics Center, Faculty of Medicine, Ain Shams University, Cairo, Egypt.

Received: 1 July 2021 Accepted: 10 August 2021

Published online: 28 September 2021

\section{References}

1. Moura RCF, Santos CA, Grecco LAC, Lazzari RD, Dumont AJ, de Duarte NC A, et al. Transcranial direct current stimulation combined with upper limb functional training in children with spastic, hemiparetic cerebral palsy: Study protocol for a randomized controlled trial. Trials. 2016;17(1):1-10. https://doi. org/10.1186/s13063-016-1534-7.

2. Horak FB. Postural orientation and equilibrium: what do we need to know about neural control of balance to prevent falls? Age Ageing. 2006; 35(suppl_2):7-11. https://doi.org/10.1093/ageing/afl077.

3. Pavão SL, dos Santos AN, Woollacott MH, Rocha NACF. Assessment of postural control in children with cerebral palsy: A review. Res Dev Disabil. 2013;34(5):1367-75. https://doi.org/10.1016/j.ridd.2013.01.034.

4. Brogren Carlberg E, Hadders-Algra M. Postural dysfunction in children with cerebral palsy: Some implications for therapeutic guidance. Neural Plast. 2005;12(2-3):221-8. https://doi.org/10.1155/NP.2005.221.

5. Hulme JB, Gallacher K, Walsh J, Niesen S, Waldron D. Behavioral and postural changes observed with use of adaptive seating by clients with multiple handicaps. Phys Ther. 1987;67(7):1060-7. https://doi.org/10.1093/ ptj/67.7.1060.

6. Chung J, Evans J, Lee C, Lee J, Rabbani Y, Roxborough L, et al. Effectiveness of adaptive seating on sitting posture and postural control in children with cerebral palsy. Pediatr Phys Ther. 2008;20(4):303-17. https://doi.org/10.1097/ PEP.0b013e31818b7bdd.

7. Piragasam GA, Rabi NM, Masnan AH. The use of sensory balls in the classroom: effects on behavioural and academic functioning of students with learning disabilities. Int J Acad Res Bus Soc Sci. 2018;8. https://doi.org/1 0.6007/ijarbss/v8-i4/4150(4).

8. Fedewa A, Davis MAC, Ahn S. Effects of stability balls on children's on-task behavior, academic achievement, and discipline referrals: a randomized controlled trial. Am J Occup Ther. 2015;69. https://doi.org/10.5014/ajot.2015. 014829(2):6902220020p1

9. Schilling DL, Schwartz IS. Alternative seating for young children with autism spectrum disorder: effects on classroom behavior. J Autism Dev Disord. 2004;34(4):423-32. https://doi.org/10.1023/B:JADD.0000037418.48587.f4.

10. Gregory DE, Dunk NM, Callaghan JP. Stability ball versus office chair: comparison of muscle activation and lumbar spine posture during prolonged sitting. Hum Factors. 2006;48(1):142-53. https://doi.org/10.1518/ 001872006776412243

11. Bagatell N, Mirigliani G, Patterson C, Reyes Y, Test L. Effectiveness of therapy ball chairs on classroom participation in children with autism spectrum 
disorders. Am J Occup Ther. 2010;64(6):895-903. https://doi.org/10.5014/a jot.2010.09149.

12. Fedewa AL, Erwin HE. Stability balls and students with attention and hyperactivity concerns: implications for on-task and in-seat behavior. Am J Occup Ther. 2011;65(4):393-9. https://doi.org/10.5014/ajot.2011.000554.

13. Schilling DL, Washington K, Billingsley FF, Deitz J. Classroom seating for children with attention deficit hyperactivity disorder: therapy balls versus chairs. Am J Occup Ther. 2003;57(5):534-41. https://doi.org/10.5014/ajot.57.5. 534.

14. Wu WL, Wang CC, Chen CH, Lai CL, Yang PC, Guo LY. Influence of therapy ball seats on attentional ability in children with attention deficit/ hyperactivity disorder. J Phys Ther Sci. 2012;24(11):1177-82. https://doi.org/1 $0.1589 /$ jpts.24.1177.

15. Al-Eisa E, Buragadda S, Melam GR. Effect of therapy ball seating on learning and sitting discomforts among saudi female students. Biomed Res Int. 2013; 2013. https://doi.org/10.1155/2013/153165:1-4.

16. Bohannon RW, Smith MB. Interrater reliability of a modified Ashworth scale of muscle spasticity. Phys Ther. 1987;67(2):206-7. https://doi.org/10.1093/ptj/ 67.2.206.

17. Van Hartingsveldt MJ, Cup EHC, Oostendorp RAB. Reliability and validity of the fine motor scale of the Peabody Developmental Motor Scales-2. Occup Ther Int. 2005:12(1):1-13. https://doi.org/10.1002/oti.11.

18. Stokes NA, Deitz JL, Crowe TK. The Peabody Developmental Fine Motor Scale: an interrater reliability study. Am J Occup Ther Off Publ Am Occup Ther Assoc. 1990;44(4):334-40. https://doi.org/10.5014/ajot.44.4.334.

19. Sakzewski L, Ziviani J, Boyd R. The relationship between unimanual capacity and bimanual performance in children with congenital hemiplegia. Dev Med Child Neurol. 2010;52(9):811-6. https://doi.org/10.1111/j.1469-8749.2 009.03588.X.

20. Brady K, Garcia T. Constraint-induced movement therapy (CIMT): Pediatric applications. Dev Disabil Res Rev. 2009;15(2):102-11. https://doi.org/10.1002/ ddrr.59.

21. Volman MJM, Wijnroks A, Vermeer A. Effect of task context on reaching performance in children with spastic hemiparesis. Clin Rehabil. 2002;16(6): 684-92. https://doi.org/10.1191/0269215502cr540oa.

22. Hanna SE, Law MC, Rosenbaum PL, King GA, Walter SD, Pollock N, et al. Development of hand function among children with cerebral palsy: growth curve analysis for ages 16 to 70 months. Dev Med Child Neurol. 2003;45(7): 448-55 Erratum in: Dev Med Child Neurol. 2003 Sep:45(9):644. PMID: 12828398.

23. Fedrizzi E, Pagliano E, Andreucci E, Oleari G. Hand function in children with hemiplegic cerebral palsy: prospective follow-up and functional outcome in adolescence. Dev Med Child Neurol. 2003;45(2):85-91. https://doi.org/1 0.1111/j.1469-8749.2003.tb00910.x.

24. Azzam AM. Effect of hand function training on improvement of hand grip strength in hemiplegic cerebral palsy in children. J Nov Physiother. 2012;02. https://doi.org/10.4172/2165-7025.1000116(06)

\section{Publisher's Note}

Springer Nature remains neutral with regard to jurisdictional claims in published maps and institutional affiliations.

\section{Submit your manuscript to a SpringerOpen ${ }^{\circ}$ journal and benefit from:}

- Convenient online submission

- Rigorous peer review

- Open access: articles freely available online

High visibility within the field

- Retaining the copyright to your article

Submit your next manuscript at $\boldsymbol{\nabla}$ springeropen.com 